\title{
Anti-GQ1b IgG antibody syndrome: clinical and immunological range
}

\author{
M Odaka, N Yuki, K Hirata
}

\begin{abstract}
Objectives-To clarify the nosological relation among Miller Fisher syndrome (MFS), Guillain-Barré syndrome (GBS) with ophthalmoplegia, Bickerstaff's brain stem encephalitis (BBE), and acute ophthalmoparesis without ataxia. Serum samples from patients with each condition often have anti-GQ1b IgG antibody.

Methods-Information on antecedent illness, initial symptoms, neurological signs during the illness, and CSF findings were reviewed in 194 patients with anti-GQ1b IgG. It was determined whether overlapping MFS and GBS (MFS/GBS), as well as overlapping $\mathrm{BBE}$ and GBS (BBE/GBS), is explained by the combined action of antiGQ1b IgG and anti-GM1 or anti-GD1a IgG, serological markers of GBS.
\end{abstract}

Results-Based on the diagnostic criteria, all the patients with acute ophthalmoparesis, MFS, MFS/GBS, BBE/GBS, and BBE had external ophthalmoplegia; all the patients with MFS, MFS/GBS, or GBS had hyporeflexia or areflexia; and all those with MFS and BBE showed ataxia. Tendon reflexes were decreased or absent in $91 \%$ of those with BBE/GBS, $67 \%$ of those with $\mathrm{BBE}$, and $53 \%$ of those with acute ophthalmoparesis. Ataxia was present in $68 \%$ of the patients with MFS/GBS and $45 \%$ of those with BBE/ GBS. Antecedent illness caused by upper respiratory tract infection had occurred in $60 \%$ to $80 \%$ of these patients, and CSF albuminocytological dissociation in $25 \%$ to $75 \%$. Anti-GM1 or anti-GD1a IgG was present in $50 \%$ of those with GBS, $35 \%$ of those with MFS/GBS, $27 \%$ of those with BBE/GBS, $16 \%$ of those with MFS, and $8 \%$ of those with BBE.

Conclusions-These findings together with the common autoantibody (antiGQ1b IgG) suggest that a common autoimmune mechanism functions in the pathogenesis of these illnesses. In a larger study, it was confirmed clinically that MFS, GBS, BBE, and acute ophthalmoparesis are closely related, forming a continuous range. This is supported by the immunological findings. The term "anti-GQ1b IgG antibody syndrome" is not intended to be used as a clinical diagnosis, but recognition of this syndrome is useful for understanding the aetiological relation among the various illnesses and for introducing the established treatments of GBS for use with other conditions. (F Neurol Neurosurg Psychiatry 2001;70:50-55)
Keywords: anti-GQ1b IgG antibody; Miller Fisher syndrome; Guillain-Barré syndrome; Bickerstaff's brain stem encephalitis; ophthalmoplegia

Miller Fisher syndrome (MFS) is characterised by the acute onset of external ophthalmoplegia, ataxia of a cerebellar type, and the loss of tendon reflexes. ${ }^{1}$ It is considered a variant of Guillain-Barré syndrome (GBS), because some patients who present with MFS progress to GBS. ${ }^{2}$ Chiba et $a l^{3}$ found that all of six patients with MFS had serum IgG antibody to GQ1b ganglioside during the acute phase of the illness, whereas none of 16 patients with GBS had it. We found anti-GQ1b IgG antibody in one of 15 patients with GBS who had impaired abduction in the right eye. ${ }^{4} \mathrm{Chiba}$ et $a \bar{P}$ subsequently detected anti-GQ1b IgG in five of six patients with GBS who showed external ophthalmoplegia, whereas none of 23 patients with GBS without ophthalmoplegia had the autoantibody. Furthermore, antiGQ1b IgG was present in all of five patients with acute postinfectious ophthalmoplegia without ataxia, "atypical MFS" in their designation. Case reports of paresis of the extraocular muscles without ataxia, which is of acute onset and self limited have been published. ${ }^{6}{ }^{7}$ Such patients usually have had antecedent infections or immunisations. We designated this condition "acute ophthalmoparesis" and reported that all of eight patients with acute ophthalmoparesis had anti-GQ1b IgG. ${ }^{8}$ The autoantibody titres in acute ophthalmoparesis did not differ significantly from those in MFS. The existence of acute ophthalmoparesis cases supports the conclusion of Chiba et at that anti-GQ1b IgG is associated closely with external ophthalmoplegia. Kusunoki et $a l^{9}$ reported that five of 149 patients with antiGQ1b IgG had ataxia without ophthalmoplegia and stated that anti-GQ1b IgG is associated with ataxia as well as ophthalmoplegia.

Murine monoclonal anti-GQ1b antibody strongly stains the paranodal regions of the extramedullary portion of human oculomotor, trochlear, and abducens nerves, and weakly stains the deep cerebellar nuclei. ${ }^{5}$ In patients with MFS those nerves were enhanced by gadolinium on MRI. ${ }^{10}$ Campylobacter jejuni, cytomegalovirus, Epstein-Barr virus, and Streptococcus pyogenes have been reported as antecedent infectious agents in MFS. ${ }^{12}$ Koga et al ${ }^{13}$ showed serological evidence of $C$ jejuni infection in $12(18 \%)$ of 65 patients with MFS, and the GQ1b epitope is present in the lipopolysaccharide of $C$ jejuni isolated from patients with MFS. ${ }^{14}$ Infection by $C$ jejuni that bears GQ1blike lipopolysaccharide may trigger production 
Table 1 Diagnostic criteria for Miller Fisher syndrome, Bickerstaff's brain stem encephalitis, and acute ophthalmoparesis

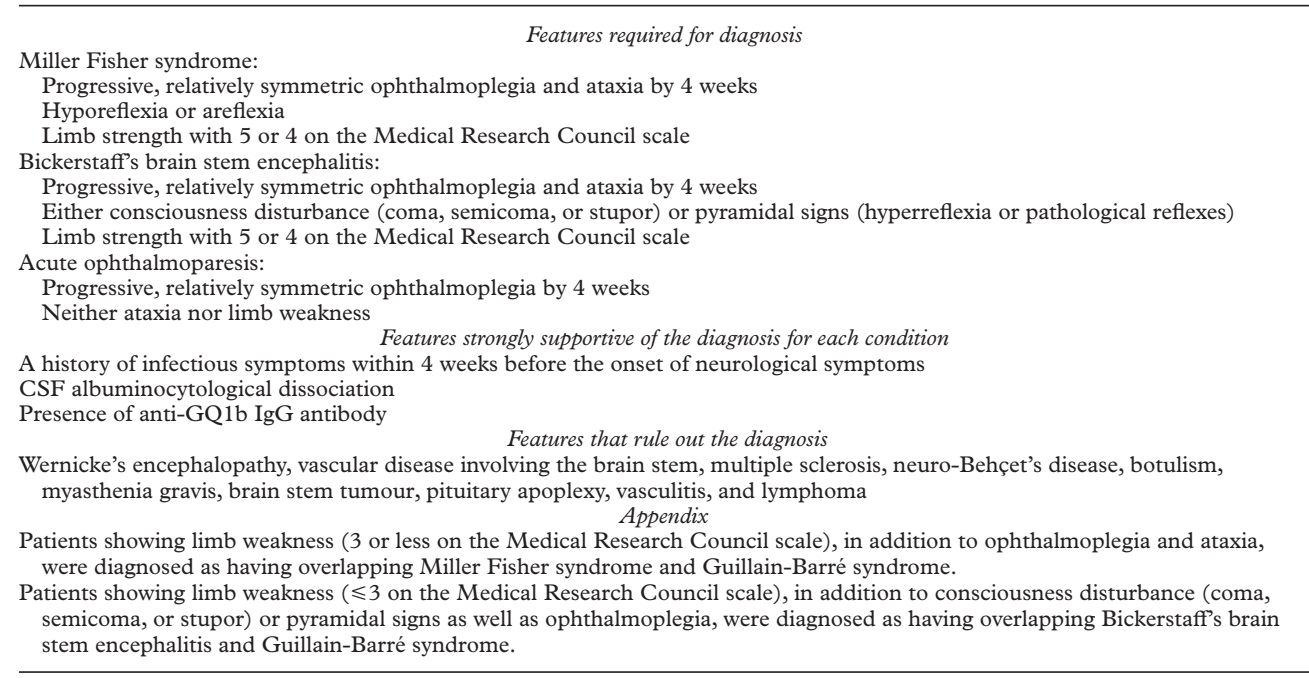

of anti-GQ1b IgG antibody in some patients with MFS, whose autoantibody may then bind to the ocular motor nerves and to the deep cerebellar nuclei causing ophthalmoplegia and cerebellar ataxia.

Bickerstaff et $a l^{15}{ }^{16}$ described patients who experienced acute onset of external ophthalmoplegia and cerebellar ataxia, a syndrome, although severe, which had a benign prognosis. Patients showing drowsiness, brisk reflexes, extensor plantar responses, and hemisensory disturbance or CSF pleocytosis usually are considered to have Bickerstaff's brain stem encephalitis (BBE) rather than MFS. Opinions differ, however, as to whether these are distinct or related conditions. We found that all of three patients with BBE had high anti-GQ1b IgG antibody titres which decreased with clinical improvement. ${ }^{17}$ The fact that BBE and MFS share common autoantibodies suggests that they are closely related, not distinct, conditions. The anti-GQ1b IgG antibody titres in BBE did not differ significantly from those in MFS. By contrast, Bickerstaff and Cloake ${ }^{15}$ speculated that the aetiology of BBE is similar to that of GBS because they detected areflexia and CSF albuminocytological dissociation. We reported necropsy findings for a patient in whom limb weakness associated with BBE had been diagnosed clinically. ${ }^{18}$ The peripheral nerves showed moderate to severe loss of large myelinated fibres. The pathological findings supported the original hypothesis by Bickerstaff and Cloake ${ }^{15}$ that BBE is closely related to GBS. That patient had anti-GQ1b IgG. Some patients with GBS have coma associated with the absence of oculocephalic reflex. ${ }^{18}$ The existence of such cases supports the hypothesis of Bickerstaff and Cloake ${ }^{15}$ that BBE is closely related to GBS.

We earlier proposed that MFS, GBS with ophthalmoplegia, acute ophthalmoparesis, and $\mathrm{BBE}$ should all be designated "anti-GQ1b IgG antibody syndrome" to clarify their nosological relations. The first aim of this larger study was to test our hypothesis that these are related conditions that form a continuous range. The second was to determine whether overlapping
MFS and GBS, as well as overlapping BBE and GBS, can be explained by the combined action of anti-GQ1b IgG with anti-GM1 or antiGD1a IgG, serological markers of GBS. ${ }^{19} 20$

\section{Materials and methods}

PATIENTS

Serum samples obtained from patients with various neurological disorders including motor neuron disease, multifocal motor neuropathy, and chronic inflammatory demyelinating polyneuropathy were referred to us from university and district general hospitals for antiganglioside antibody assays. Serum antibodies to GM1, GM2, GD1a, GD1b, GT1b, and GQ1b were measured using the enzyme linked immunosorbent assay (ELISA) described elsewhere. ${ }^{21}$ Serum was considered positive when the antibody titre was 500 or more. Anti-GQ1b IgG antibody was positive in the samples from 245 patients tested between December 1994 and September 1998. Medical records of the patients were reviewed by one of us (MO). Information on antecedent illness, initial symptoms, neurological signs during the illness, and CSF findings were obtained from 194 patients. The following signs were assessed: consciousness disturbance; blepharoptosis, internal or external ophthalmoplegia, nystagmus, facial weakness, and bulbar palsy; limb weakness ( $\leqslant 3$ on the Medical Research Council scale); Babinski's sign; deep tendon reflexes (brisk, normal, decreased, or absent); truncal or limb ataxia; and deep or superficial sense impairment. In the present study, the albuminocytological dissociation was defined as high CSF protein ( $\geqslant 45 \mathrm{mg} / \mathrm{dl})$ with normal cellularity $(\leqslant 5 / \mu 1)$.

\section{DIAGNOSTIC CRITERIA}

The diagnosis of GBS was based on the established clinical criteria, and extensor plantar responses do not exclude the diagnosis if other features are typical. ${ }^{22}$ The diagnostic criteria used for MFS, BBE, and acute ophthalmoparesis are given in table 1. Patients who showed limb weakness, in addition to ophthalmoparesis and ataxia, were considered to have 
Table 2 Clinical profiles of patients with anti-GQ1b IgG antibody

\begin{tabular}{|c|c|c|c|c|c|c|c|c|}
\hline & Total & $\begin{array}{l}\text { Acute } \\
\text { ophthalmoparesis }\end{array}$ & $M F S$ & $M F S / G B S$ & $G B S$ & $B B E / G B S$ & $B B E$ & Unclassified \\
\hline Patients (n) & 194 & 15 & 110 & 31 & 8 & 11 & 12 & 7 \\
\hline Sex (men/women) & $106 / 88$ & $6 / 9$ & $66 / 44$ & $16 / 15$ & $4 / 4$ & $5 / 6$ & $5 / 7$ & $4 / 3$ \\
\hline Median age (range) & $37(2-78)$ & $30(9-69)$ & $41(2-78)$ & $33(6-76)$ & $36(17-50)$ & $42(24-76)$ & $37(27-69)$ & $31(18-66)$ \\
\hline Antecedent illness: & $(\%)$ & $(\%)$ & $(\%)$ & (\%) & $(\%)$ & $(\%)$ & $(\%)$ & $(\%)$ \\
\hline Upper respiratory infection & 80 & 60 & 86 & 74 & 63 & 82 & 75 & 71 \\
\hline Diarrhoea & 14 & 27 & 10 & 19 & 63 & 0 & 0 & 14 \\
\hline \multicolumn{9}{|l|}{ Initial symptoms: } \\
\hline Diplopia & 67 & 100 & 75 & 39 & 0 & 55 & 75 & 71 \\
\hline Gait disturbance & 29 & 0 & 32 & 42 & 75 & 18 & 33 & 43 \\
\hline Limb weakness & 12 & 0 & 3 & 35 & 100 & 9 & 0 & 14 \\
\hline Photophobia & 8 & 0 & 10 & 10 & 0 & 0 & 0 & 14 \\
\hline Dysarthria & 7 & 7 & 4 & 16 & 0 & 18 & 8 & 14 \\
\hline Dysphagia & 2 & 0 & 2 & 3 & 0 & 0 & 0 & 0 \\
\hline Dysaesthesia & 4 & 0 & 3 & 10 & 13 & 9 & 0 & 0 \\
\hline Blepharoptosis & 2 & 0 & 3 & 0 & 0 & 0 & 8 & 0 \\
\hline Consciousness disturbance & 1 & 0 & 0 & 0 & 0 & 9 & 8 & 0 \\
\hline \multicolumn{9}{|c|}{ Neurological signs during the course of the illness: } \\
\hline \multicolumn{9}{|c|}{ Consciousness disturbance } \\
\hline Drowsiness & 7 & 0 & 3 & 13 & 0 & 36 & 17 & 0 \\
\hline Stupor, semicoma, coma & 4 & 0 & 0 & 0 & 0 & 27 & 33 & 0 \\
\hline Blepharoptosis & 45 & 40 & 51 & 42 & 0 & 36 & 17 & 29 \\
\hline External ophthalmoplegia & 91 & 100 & 100 & 100 & 0 & 100 & 100 & 100 \\
\hline Internal ophthalmoplegia & 36 & 7 & 37 & 52 & 0 & 27 & 42 & 29 \\
\hline Nystagmus & 22 & 27 & 16 & 29 & 25 & 18 & 33 & 57 \\
\hline Facial weakness & 34 & 13 & 23 & 71 & 13 & 82 & 33 & 14 \\
\hline Bulbar palsy & 29 & 13 & 20 & 61 & 13 & 82 & 25 & 14 \\
\hline Limb weakness $\star$ & 26 & 0 & 0 & 100 & 100 & 100 & 0 & 14 \\
\hline \multicolumn{9}{|l|}{ Tendon reflex } \\
\hline Brisk & 4 & 0 & 0 & 0 & 0 & 9 & 33 & 0 \\
\hline Normal & 6 & 47 & 0 & 0 & 0 & 0 & 0 & 100 \\
\hline Decreased & 30 & 33 & 29 & 32 & 62 & 35 & 25 & 0 \\
\hline Absent & 60 & 20 & 71 & 68 & 38 & 56 & 42 & 0 \\
\hline Babinski’s sign & 8 & 0 & 0 & 0 & 0 & 64 & 67 & 0 \\
\hline Ataxia & 80 & 0 & 100 & 68 & 0 & 45 & 100 & 100 \\
\hline Deep sense impairment & 26 & 0 & 27 & 39 & 13 & 55 & 8 & 29 \\
\hline Superficial sense impairment & 35 & 20 & 36 & 42 & 38 & 36 & 8 & 29 \\
\hline CSF albuminocytological dissociation & 58 & 25 & 66 & 43 & 25 & 75 & 42 & 80 \\
\hline
\end{tabular}

MFS= Miller Fisher syndrome; GBS=Guillain-Barré syndrome; BBE=Bickerstaff's brain stem encephalitis; MFS/GBS=overlapping Miller Fisher syndrome and Guillain-Barré syndrome; BBE/GBS=overlapping Bickerstaff’s brain stem encephalitis and Guillain-Barré syndrome.

$\star 3$ or less on the Medical Research Council scale

overlapping MFS and GBS (MFS/GBS). Patients who showed limb weakness, in addition to consciousness disturbance (coma, semicoma, or stupor) or pyramidal sign as well as ophthalmoparesis and ataxia, were considered to have overlapping BBE and GBS (BBE/ GBS).

STATISTICAL ANALYSIS

Analyses were performed with Stat View Version 4.5-J software (Abacus Concepts, Berkeley, CA, USA). Differences in proportions were tested by the $\chi^{2}$ test or Fisher's exact test (two tailed). A p value $<0.05$ was used for significance in all the comparisons.

\section{Results}

CLINICAL FEATURES OF ANTI-GQ1B IGG ANTIBODY SYNDROME

Clinical profiles of the 194 patients (median age $37 ; 106$ men; 88 women) are shown in table 2. Ninety four per cent had antecedent illnesses (upper respiratory tract infection alone $75 \%$; diarrhoea alone $9 \%$; both upper respiratory tract infection and diarrhoea 5\%). As initial symptoms respectively, 130 patients $(67 \%)$ and 56 patients $(29 \%)$ complained of diplopia and gait disturbance, and 25 patients $(13 \%)$ had both. Consciousness was disturbed in $11 \%$ (drowsiness $7 \%$; stuporous or comatose $4 \%$ ). External ophthalmoplegia was present in $91 \%$ and ataxia in $80 \%$ (both truncal and limb 50\%; truncal alone $20 \%$; limb alone $10 \%$ ). Of 155 patients who had ataxia, $41(26 \%)$ had deep sense impairment. Limb weakness was appreciable in $26 \%$ of the 194 patients. Deep tendon reflexes were absent in $60 \%$ of them, decreased in $30 \%$, normal in $6 \%$, and brisk in $4 \%$. Extensor plantar response was present in $8 \%$, and hyperreflexia or the Babinski's sign in $9 \%$. Patients with external ophthalmoplegia had associated ataxia $(p<0.0001$; odds ratio 33.7; 95\% confidence interval 8.1-139.6), whereas neither ophthalmoplegia nor ataxia were associated with hyporeflexia or areflexia $(p=0.8$ and $p=0.5$ respectively). Fifty eight per cent had CSF albuminocytological dissociation.

CLINICAL FEATURES OF EACH DIAGNOSTIC CONDITION

The diagnosis of MFS was made for 110 (57\%), MFS/GBS for $31(16 \%)$, acute ophthalmoparesis for $15(8 \%), \mathrm{BBE}$ for $12(6 \%)$, $\mathrm{BBE} / \mathrm{GBS}$ for $11(6 \%)$, and GBS for eight $(4 \%)$. The illnesses of the other seven $(4 \%)$ were unclassified. Among the patients included in this study, illustrative cases of acute ophthalmoparesis, ${ }^{23} \mathrm{MFS},{ }^{10} \mathrm{BBE},{ }^{24}$ and $\mathrm{MFS} / \mathrm{GBS}^{25}$ have been reported previously. An illustrative case of $\mathrm{BBE} / \mathrm{GBS}$ is described below.

\section{Case report of $B B E / G B S$}

A 24 year old man had a cough and nasal obstruction which abated over a few days. One week after resolution of this illness, he experienced diplopia and numbness of the arms (day 1 ). On day 2 , he was unable to stand. He became drowsy with dysarthria, and was 
Table 3 Antiganglioside IgG antibodies in each condition

\begin{tabular}{|c|c|c|c|c|c|c|c|}
\hline \multirow[b]{2}{*}{ Diagnosis } & \multirow[b]{2}{*}{ No } & \multicolumn{6}{|c|}{ IgG antibodies to: } \\
\hline & & $\begin{array}{l}G M 2 \\
(\%)\end{array}$ & $\begin{array}{l}G M 1 \\
(\%)\end{array}$ & $\begin{array}{l}\text { GD1a } \\
(\%)\end{array}$ & $\begin{array}{l}G D 1 b \\
(\%)\end{array}$ & $\begin{array}{l}\text { GT1b } \\
(\%)\end{array}$ & $\begin{array}{l}\text { GM1 or } G D 1 a \\
(\%)\end{array}$ \\
\hline Acute ophthalmoparesis & 15 & 0 & 0 & 0 & 7 & 0 & 0 \\
\hline MFS & 110 & 1 & 5 & 13 & 15 & 2 & 16 \\
\hline MFS/GBS & 31 & 10 & 16 & 29 & 35 & 13 & 35 \\
\hline GBS & 8 & 25 & 38 & 50 & 50 & 50 & 50 \\
\hline $\mathrm{BBE} / \mathrm{GBS}$ & 11 & 9 & 0 & 27 & 36 & 27 & 27 \\
\hline BBE & 12 & 0 & 8 & 8 & 17 & 0 & 8 \\
\hline Unclassified & 7 & 0 & 0 & 14 & 14 & 14 & 0 \\
\hline
\end{tabular}

Abbreviations as for table 2 . and $25 \%$ of those with acute ophthalmoparesis and GBS.

ANTI-GM1 OR ANTI-GD1a IgG IN EACH DIAGNOSTIC CONDITION

Table 3 shows the immunological range for all the conditions. Anti-GM1 IgG antibody was present in $38 \%$ of the patients with GBS, $16 \%$ of those with MFS/GBS, $8 \%$ of those with $\mathrm{BBE}$, and $5 \%$ of those with MFS. Anti-GD1a IgG antibody was present in $50 \%$ of the patients with GBS, $29 \%$ of those with MFS/ GBS, $27 \%$ of those with BBE/GBS, $13 \%$ of those with MFS, and $8 \%$ of those with BBE. Anti-GM1 or anti-GD1a IgG antibody was present in $50 \%$ of the patients with GBS, 35\% of those with $\mathrm{MFS} / \mathrm{GBS}, 27 \%$ of those with $\mathrm{BBE} / \mathrm{GBS}, 16 \%$ of those with MFS, and $8 \%$ of those with BBE. Neither anti-GM1 nor anti-GD1a IgG was present in acute ophthalmoparesis.

scale. Deep tendon reflexes were absent, but extensor plantar responses remained. Finger to nose and heel to knee tests showed dysmetria and decomposition. Hypaesthesia of the glove and stocking type was present. Protein in CSF was $27 \mathrm{mg} / \mathrm{dl}$ with 1 cell $/ \mu \mathrm{l}$ on day 7 , and 295 $\mathrm{mg} / \mathrm{dl}$ with 6 cells/ $\mu \mathrm{l}$ on day 14 . Seven sessions of immunoadsorption therapy were given on days $3,4,5,7,9,11$, and 13 . In Japan, the patients with GBS and related disorders often receive immunoadsorption using a tryptophan conjugated column (TR-350, Asahi Medical, Japan) which highly absorbs anti-GQ1b IgG. ${ }^{26}$ On day 6 , he began to recover consciousness, and limb weakness diminished. He could walk without support on day 19, and diplopia disappeared on day 39.

Table 2 shows the antecedent illness, initial symptoms, and neurological signs for each condition. For antecedent illness, the frequency of upper respiratory tract infection was much higher than that of diarrhoea for all the conditions except GBS. As the initial symptom, diplopia was experienced in $100 \%$ of the patients with acute ophthalmoparesis, $75 \%$ of those with both MFS and BBE, 55\% of those with BBE/GBS, and $39 \%$ of those with MFS/GBS. Gait disturbance was experienced by $75 \%$ of the patients with GBS, $42 \%$ of those with MFS/GBS, $33 \%$ of those with BBE, 32\% of those with MFS, and $18 \%$ of those with $\mathrm{BBE} / \mathrm{GBS}$. On the basis of the diagnostic criteria, all the patients with acute ophthalmoparesis, MFS, MFS/GBS, BBE/GBS, and BBE had external ophthalmoplegia; all the patients with MFS, MFS/GBS, and GBS had hyporeflexia or areflexia; and all the patients with MFS and $\mathrm{BBE}$ had ataxia. Tendon reflexes were decreased or absent in $91 \%$ of those with BBE/GBS, $67 \%$ of those with BBE, and $53 \%$ of those with acute ophthalmoparesis. Ataxia was present in $68 \%$ of the patients with MFS/GBS and $45 \%$ of those with BBE/GBS. Consciousness was altered in $63 \%$ of the patients with $\mathrm{BBE} / \mathrm{GBS}, 50 \%$ of those with $\mathrm{BBE}, 13 \%$ of those with MFS/GBS, and 3\% of those with MFS. Albuminocytological dissociation in CSF was present in $75 \%$ of the patients with BBE/GBS, $66 \%$ of those with MFS, $43 \%$ of those with $\mathrm{MFS} / \mathrm{GBS}, 42 \%$ of those with $\mathrm{BBE}$,

\section{Discussion}

OUR DIAGNOSTIC CRITERIA FOR MFS AND BBE One of the three patients originally described by Fisher ${ }^{1}$ showed mild drowsiness. Drowsiness therefore may accompany both MFS and BBE according to the criteria used in this study. All of the three cases reported by Fisher fulfilled our criteria for MFS, but not for BBE, whereas six of eight cases described by Bickerstaff $^{16}$ fulfilled our criteria for BBE, but not for MFS. In case 4 reported by Bickerstaff ${ }^{16}$ the patient showed gross flaccid weakness. That condition was diagnosed as BBE/GBS based on our criteria. Because another patient (case 6) did not show ophthalmoplegia, neither the criteria for MFS nor BBE were fulfilled.

Al-Din et $a l^{27}$ (with Bickerstaff) reported 18 patients with BBE (11 with drowsiness, one with coma). Tendon reflexes were absent in 11 of them, normal in three, and brisk in four. Babinski's sign was positive in five patients, and deep tendon reflexes were normal in one and absent in one. Ropper ${ }^{28}$ criticised this report and considered that six of the cases were typical MFS and the other 12 obscure brain stem lesions without peripheral neuropathy. Based on our criteria, MFS would have been diagnosed in six cases (cases 3, 5, 11, 13, 15, and 16) and $\mathrm{BBE}$ in 12. Our criteria therefore should prove useful for making clinical diagnoses of MFS and BBE. Later, Al-Din et al ${ }^{9}$ (without Bickerstaff) diagnosed MFS in three comatose patients who showed ophthalmoplegia, ataxia, and areflexia. Patients 2 and 3 showed tetraplegia. Based on our criteria, case 1 in their report would be BBE and cases 2 and $3 \mathrm{BBE} / \mathrm{GBS}$. Among our patients primary physicians had diagnosed four cases of "MFS associated with hyperreflexia" which, in this study, were diagnosed as BBE.

CLINICALLY CONTINUOUS RANGE OF MFS, GBS, BBE, AND ACUTE OPHTHALMOPARESIS

One hundred and forty two (73\%) of the 194 patients with anti-GQ1b IgG fulfilled the criteria for the syndrome of ophthalmoplegia, ataxia, and areflexia according to Al-Din et $a l^{30}$; whereas 110 had MFS, $21 \mathrm{MFS} / \mathrm{GBS}$, six 
$\mathrm{BBE}$, and five $\mathrm{BBE} / \mathrm{GBS}$ according to our criteria. All the patients with MFS fulfilled the criteria for the syndrome of ophthalmoplegia, ataxia, and areflexia, whereas only $68 \%$ of those with MFS/GBS, $50 \%$ of those with BBE, and $45 \%$ of those with BBE/GBS did. These findings indicate considerable overlapping of the syndrome of ophthalmoplegia, ataxia, and areflexia and anti-GQ1b IgG syndrome. The patients with anti-GQ1b IgG, however, had a statistically significant correlation only between ophthalmoplegia and ataxia. Neither ophthalmoplegia nor ataxia was correlated with areflexia. This is further support for the earlier report that ophthalmoplegia and ataxia are associated closely with anti-GQ1b IgG. $^{5}{ }^{9}$ Anti-GQ1b IgG antibody syndrome comprises a wide range presenting with ophthalmoplegia and ataxia. Chemical analysis showed that the optic nerves and the oculomotor, trochlear, and abducens nerves had similar concentration of GQ1b. ${ }^{31}$ The GQ1b epitope localisation suggested immunohistochemically, however, was different: no accumulation of staining in the specific region of the optic nerve. Their findings could explain why none of our patients who had anti-GQ1b IgG antibody showed optic neuropathy.

Seven patients were unclassified by the criteria we used. They had ophthalmoplegia and ataxia, but their tendon reflexes were normal during the illness. We elsewhere reported on four patients with Campylobacter neuropathy associated with anti-GM1 IgG, in whom muscle stretch reflexes were preserved or hyperactive throughout the illness. ${ }^{32}$ Although loss of tendon jerks is a valuable diagnostic feature of GBS, the presence of such unclassified cases suggests that the diagnostic criteria should be extended because this loss also could apply to MFS; therefore, those cases could be diagnosed as MFS. The other cases could be diagnosed as BBE because three of the 18 patients reported by Al-Din et $a l^{27}$ showed normal muscle stretch reflexes. Furthermore, the seven unclassified cases could be considered as overlapping MFS and BBE. The existence of such cases also supports the hypothesis that these conditions form a continuous range and that ophthalmoplegia and ataxia are more important than areflexia in that range. Thirty one cases of $\mathrm{MFS} / \mathrm{GBS}$ and 11 cases of $\mathrm{BBE} / \mathrm{GBS}$ were reported in this study. The existence of

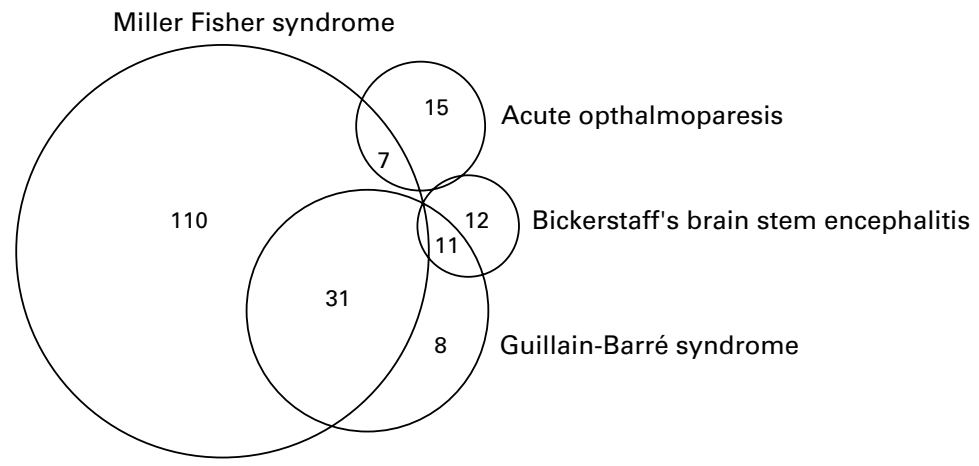

Nosological relations between Miller Fisher syndrome, acute ophthalmoparesis, Bickerstaff's brain stem encephalitis, and Guillain-Barré syndrome. these overlapping cases strongly supports the idea of continuity between MFS and GBS, as well as between BBE and GBS. ${ }^{2} 18$

Double vision was an initial symptom in three of four of the patients with both MFS and $\mathrm{BBE}$, and gait disturbance in one of three. Some patients who have MFS or BBE may not show ataxia at the onset of neurological symptoms and therefore acute ophthalmoparesis should be diagnosed. This suggests that acute ophthalmoparesis without ataxia is a restricted and mild condition of MFS or BBE.

IMMUNOLOGICAL EVIDENCE THAT MFS, GBS, BBE, AND ACUTE OPHTHALMOPARESIS FORM A CONTINUOUS RANGE

For each condition, antecedent illness caused by upper respiratory infection was present in $60 \%$ to $80 \%$ of the patients, and CSF albuminocytological dissociation in $25 \%$ to $75 \%$. This suggests that a common autoimmune mechanism functions in the pathogenesis of these conditions.

Whereas anti-GQ1b IgG is an immunological marker of MFS and related disorders, ${ }^{3-5} 8$ anti-GM1 IgG autoantibody may be a serological marker of axonal GBS. ${ }^{19}{ }^{33}$ Anti-GD1a IgG antibody is closely associated with the axonal form of GBS, ${ }^{20}$ but was not detected in typical patients with MFS who did not show limb weakness. ${ }^{34}{ }^{34} C$ jejuni is a common antecedent agent in MFS, ${ }^{13}$ as well as in GBS, ${ }^{35}{ }^{36}$ and some patients who present as having MFS subsequent to $C$ jejuni enteritis then progress to GBS. ${ }^{3738} \mathrm{~A}$ single strain of $C$ jejuni has several lipopolysaccharides that bear epitopes common to such gangliosides as GM1, GD1a, and GQ1b. ${ }^{39}{ }^{40}$ Infection by a strain of $C$. jejuni may induce not only anti-GQ1b IgG, but anti-GM1 or anti-GD1a IgG in some patients with MFS/ GBS. Among our patients with MFS/GBS, $C$ jejuni actually was isolated from one with MFS/GBS who had anti-GQ1b and antiGDla IgG antibodies (case 4 of Ohtsuka et $a l^{41}$ ). We therefore compared the frequencies of anti-GM1 or anti-GD1a IgG in MFS, MFS/ GBS, and GBS. As expected, the frequency of anti-GM1 or anti-GD1a IgG in MFS/GBS (35\%) was between that in MFS (16\%) and that in GBS ( $50 \%)$. Moreover, the frequency of anti-GM1 or anti-GD1a IgG in BBE/GBS $(27 \%)$ was between that in $\mathrm{BBE}(8 \%)$ and that in GBS (50\%). In addition to the similarities in clinical presentation and share of anti-GQ1b IgG autoantibody, immunological results for the overlapping cases show that MFS, GBS, and $\mathrm{BBE}$ are closely related, not distinct, autoimmune mediated disorders. The patients with acute ophthalmoparesis had neither antiGM1 nor anti-GD1a IgG. Serologically this indicates that MFS or BBE is located between acute ophthalmoparesis and GBS.

RECOGNITION OF ANTI-GQ1b IgG ANTIBODY SYNDROME AND ITS THERAPY

In this larger study, we confirmed clinically that MFS, GBS, BBE, and acute ophthalmoparesis are closely related and form a continuous range (figure). This was supported by immunological findings. We do not intend the term "anti- 
GQ1b IgG antibody syndrome" to be used as a clinical diagnosis, but recognition of this syndrome is useful for understanding the aetiological relations of MFS, GBS, BBE, and acute ophthalmoparesis. Moreover, established treatments for GBS (plasmapheresis and intravenous immunoglobulin) may be more readily introduced as treatments for BBE and acute ophthalmoparesis, as well as for MFS. Some patients with BBE and acute ophthalmoparesis have responded favourably to plasmapheresis and intravenous immunoglobulin ${ }^{2324} 42 \mathrm{Re}-$ cent studies by Buchwald et $a t^{43}$ and Plomp et $a l^{44}$ show that anti-GQ1b IgG, at least, is a pathophysiological mediator of motor symptoms in MFS and related disorders. This is theoretical evidence that removal of the autoantibody is efficacious treatment for patients with anti-GQ1b IgG.

This research was supported in part by grants in aid for Scientific Research (10780482 and 10557063 to NY) from the Ministry of Education, Science, Culture, and Sports of Japan and istry of Education, Science, Culture, and Sports of Japan and Research Grant for Neuroimmunological Diseases from the Ministry of Health and Welfare of Japan.

1 Fisher M. An unusual variant of acute idiopathic polyneuritis: syndrome of ophthalmoplegia, ataxia, and areflexia. $N$ tis: syndrome of ophthalmop.
Engl $\mathcal{F}$ Med 1956;255:57-65.

Engl 7 Med 1956;255:57-65.
2 Arnason BGW, Soliven B. Acute inflammatory demyelinating polyradiculoneuropathy. In: Dyck PJ, Thomas PK, Griffin JW, et al, eds. Peripheral neuropathy, 3rd ed. Philadelphia: Saunders, 1993:1437-97.

3 Chiba A, Kusunoki S, Shimizu T, et al. Serum IgG antibody to ganglioside GQ1b is a possible marker of Miller Fisher syndrome. Ann Neurol 1992;31:677-9.

4 Yuki N, Sato S, Tsuji S, et al. Frequent presence of anti-GQ1b antibody in Fisher's syndrome. Neurology 1993; 43:414-7.

5 Chiba A, Kusunoki S, Obata H, et al. Serum anti-GQ1b IgG antibody is associated with ophthalmoplegia in Miller Fisher syndrome and Guillain-Barré syndrome: clinical and immunohistochemical studies. Neurology 1993;43: 1911-7.

6 Slavin ML. Gaze palsy associated with viral syndrome. $A m \mathcal{F}$ Ophthalmol 1985;100:468-73.

7 v d Kruijk RA, Lampe AS, Endtz HP. Bilateral abducens paresis following Campylobacter jejuni enteritis. I Infect 292; $24: 215-6$

8 Yuki N. Acute paresis of extra ocular muscles associated with IgG anti-GQ1b antibody. Ann Neurol 1996;39:66872 .

9 Kusunoki S, Chiba A, Kanazawa I. Anti-GQ1b IgG antibody is associated with ataxia as well as ophthalmoplegia. Muscle Nerve 1999;22:1071-4

10 Nagaoka U, Kato T, Kurita K, et al. Cranial nerve enhancement on three-dimensional MRI in Miller Fisher syndrome. Neurology 1996;47:1601-2.

11 Tanaka H, Yuki N, Hirata K. Trochlear nerve enhancement on three-dimensional magnetic resonance imaging in Fisher syndrome. Am f Ophthalmol 1998;126:322-4.

12 Yuki N, Hirata K. Fisher's syndrome and group A streptococcal infection. F Neurol Sci 1998;160:64-6.

13 Koga M, Yuki N, Takahashi $M$, et al. Close association of IgA anti-ganglioside antibodies with antecedent Campylobacter jejuni infection in Guillain-Barré and Fisher's bacter jejuni infection in Guillain-Barre

14 Yuki N, Taki T, Takahashi M, et al. Molecular mimicry between GQ1b ganglioside and lipopolysaccharides of Campylobacter jejuni isolated from patients with Fisher's syndrome. Ann Neurol 1994;36:791-3.

15 Bickerstaff ER, Cloake PCP. Mesencephalitis and rhombencephalitis. $B M \mathcal{F} 1951 ;$ ii: $77-81$.

16 Bickerstaff ER. Brain-stem encephalitis. further observations on a grave syndrome with benign prognosis. $B M f$ $1957 ; \mathrm{i}: 1384-7$

17 Yuki N, Sato S, Tsuji S, et al. An immunologic abnormality common to Bickerstaff's brain stem encephalitis and Fisher's syndrome. F Neurol Sci 1993;118:83-7.

18 Yuki N, Wakabayashi K, Yamada $M$, et al. Overlap of Guillain-Barré syndrome and Bickerstaff's brain stem encephalitis. F Neurol Sci 1997;145:119-21.
19 Kornberg AJ, Pestronk A, Bieser K, et al. The clinical correlates of high-titer IgG anti-GM1 antibodies. Ann Neurol 1994;35:234-7.

20 Ho TW, Willison HJ, Nachamkin I, et al. Anti-GD1a antibody is associated with axonal but not demyelinating forms of Guillain-Barré syndrome. Ann Neurol 1999;45: 168-73.

21 Odaka $\mathrm{M}$, Yuki $\mathrm{N}$, Yoshino $\mathrm{H}$, et al. $\mathrm{N}$-glycolylneuraminic acid-containing GM1 is a new molecule for serum antibody in Guillain-Barré syndrome. Ann Neurol 1998;43: 829-34.

22 Asbury AK, Cornblath DR. Assessment of current diagnostic criteria for Guillain-Barré syndrome. Ann Neurol 1990; 27(suppl):S21-4.

23 Kikuchi M, Tagawa Y, Saotome S, et al. Acute ophthalmoparesis associated with IgG anti-GQ1b antibody subsequent to Streptococcus pyogenes infection. Eur $\mathcal{F}$ Paediatr Neurol 1997;1:47-8

24 Kikuchi $\mathrm{M}$, Tagawa $\mathrm{Y}$, Iwamoto $\mathrm{H}$, et al. Bickerstaff's brain stem encephalitis associated with IgG anti-GQ1b antibody subsequent to Mycoplasma pneumoniae infection: favorable response to immunoadsorption therapy. 7 Child Neurol 1997; 12:403-5.

25 Odaka M, Yuki N, Hirata K. Bilateral ballism in a patient with overlapping Fisher's and Guillain-Barré syndromes. $\mathcal{f}$ Neurol Neurosurg Psychiatry 1999;67:206-8.

26 Yuki N. Tryptophan-immobilized column adsorbs immunoglobulin G anti-GQ1b antibody from Fisher's syndrome: a new approach to treatment. Neurology 1996;46:1644-51.

27 Al-Din AN, Anderson M, Bickerstaff ER, et al. Brainstem encephalitis and the syndrome of Miller Fisher: a clinical study. Brain 1982;105:481-95.

28 Ropper AH. The CNS in Guillain-Barré syndrome. Arch Neurol 1983;40:397-8.

29 Al-Din ASN, Jamil AS, Shakir R. Coma and brain stem areflexia in brain stem encephalitis (Fisher's syndrome). BMF 1985;24:535-6.

30 Al-Din SN, Anderson M, Eeg-Olofsson O, et al. Neuroophthalmic manifestations of the syndrome of ophthalmoplegia, ataxia and areflexia: a review. Acta Neurol Scand 1994;89:157-63.

31 Chiba A, Kusunoki S, Obata H, et al. Ganglioside composition of the human cranial nerves, with special reference to pathophysiology of Miller Fisher syndrome. Brain Res 1997;743:32-6.

32 Yuki N, Hirata K. Preserved tendon reflexes in Campylobacter neuropathy [letter]. Ann Neurol 1998;43:546-7.

33 Kuwabara S, Yuki N, Koga M, et al. IgG anti-GM1 antibody is associated with reversible conduction failure and axonal degeneration in Guillain-Barré syndrome. Ann Neurol 1998;44:202-8.

34 Willison HJ, Veitch J, Paterson G, et al. Miller Fisher syndrome is associated with serum antibodies to GQ1b ganglioside, $\mathcal{F}$ Neurol Neurosurg Psychiatry 1993;56:204-6.

35 Rees JH, Gregson NA, Hughes RA. Anti-ganglioside GM1 antibodies in Guillain-Barré syndrome and their relationship to Campylobacter jejuni infection. Ann Neurol 38.809-16.

36 Jacobs BC, Rothbarth PH, van der Meché FG, et al. The spectrum of antecedent infections in Guillain-Barré syndrome: a case-control study. Neurology 1998;51:1110-5.

37 Constant OC, Bentley CC, Denman AM, et al. The Guillain-Barré syndrome following Campylobacter enteritis with recovery after plasmapheresis. F Infect 1983;6:8991.

38 Yuki N, Takahashi $\mathrm{M}$, Tagawa $\mathrm{Y}$, et al. Association of Campylobacter jejuni serotype with antiganglioside antibody in Guillain-Barré syndrome and Fisher's syndrome. Ann Neurol 1997;42:28-33.

39 Aspinall GO, Fujimoto S, McDonald AG, et al. Lipopolysaccharides from Campylobacter jejuni associated with Guillain-Barré syndrome patients mimic human gangliosides in structure. Infect Immun 1994;62:2122-5.

40 Yuki N, Ichihashi Y, Taki T. Subclass of IgG antibody to GM1 epitope-bearing lipopolysaccharide of Campylobacter jejuni in patients with Guillain-Barré syndrome. $\mathcal{F}$ Neuroimmunol 1995;60:161-4.

41 Ohtsuka K, Nakamura Y, Hashimoto M, et al. Fisher syndrome associated with IgG anti-GQ1b antibody following infection by a specific serotype of Campylobacter ing infection by a specific serotype

42 Yuki N. Successful plasmapheresis in Bickerstaff's brain stem encephalitis associated with anti-GQ1b antibody. $\mathcal{F}$ Neurol Sci 1995;131:108-10.

43 Buchwald B, Toyka KV, Zielasek J, et al. Neuromuscular blockade by IgG antibodies from patients with GuillainBarré syndrome: a macro-patch-clamp study. Ann Neurol 1998;44:913-22.

44 Plomp JJ, Molenaar PC, O'Hanlon GM, et al. Miller Fisher anti-GQ1b antibodies: $\alpha$-latrotoxin-like effects on motor end plates. Ann Neurol 1999;45:189-99. 